\title{
RIAD. La experiencia de los PIME's aplicada a un aprendizaje basado en proyectos
}

Maite Palomares Figueres, Ana Portalés Mañanos, Chele Esteve Sendra, María Vargas Colas, $M^{\mathrm{a}}$ Teresa Sebastiá, $\mathbf{M}^{\mathrm{a}}$ Eugenia Babiloni Griñón, Nuria Pascual Seva, Sabina Asensio Rueda

Maite Palomares Figueres (Depto. Composición Arquitectónica, ETSA UPV, mapafi@cpa.upv.es); Ana Portalés Mañanos (Dpto. Urbanismo, ETSA UPV, anporma@urb.upv.es; Chele Esteve Sendra (Dpto. Dibujo, maessen@dib.upv.es); MariaVargas Colas (Dpto. Tecnología de Alimentos mavarco@tal.upv.es); Sabina Asensio Rueda (Proyectos de Ingeniería, sasensio@dpi.upv.es; $\mathrm{M}^{\mathrm{a}}$ Teresa Sebastiá (Dpto. Ingeniería Hidráulica, mtsebastia@hma.upv.es); Ma Eugenia Babiloni Griñón (Organización de Empresas, UPV, mabagri@doe.upv.es); Nuria Pascual Seva (Dpto. Producción Vegetal, nupasse@prv.upv.es)

\begin{abstract}
Resumen
RIAD (2010) es un equipo de innovación docente, de carácter interdisciplinar, cuyo objetivo es trabajar competencias transversales entre distintas titulaciones a partir de un modelo de enseñanza-aprendizaje basado en proyectos, favorecido por el contexto de una U.P. que favorece el intercambio entre profesores y alumnos de distintas disciplinas con objetivos comunes. Desarrollado mediante una metodología de aprendizaje basado en proyectos en equipo, propicia la aparición de sinergias y una formación universitaria próxima a la práctica profesional. Se diseñó un proyecto que aglutinara las peculiaridades de las distintas disciplinas: el diseño de una bodega, con posibilidades de realización. Se implementaron sucesivos proyectos de innovación y mejora educativa (PIME) como estrategia docente.
\end{abstract}

(2010/2011): “desarrollo de una metodología de enseñanza-aprendizaje orientada hacia la adquisición de competencias mediante el desarrollo de proyectos multidisciplinares".

(2011/2012): “desarrollo y evaluación de competencias transversales mediante metodologías de enseñanza-aprendizaje interdisciplinares”. Las tareas derivadas de dicho PIME promovieron la aparición de un blog como foro de interacción entre profesores y alumnos, siendo el germen para el que se realizó durante el curso PIME 2012/2013 "El blog como portafolio virtual de un proyecto interdisciplinar". 
RIAD. La experiencia de los PIME's aplicada a un aprendizaje basado en proyectos

Palabras clave: innovación docente, convergencia, competencias, metodología, evaluación, blog, sinergias, proyectos, bodegas

\section{Introducción}

Este artículo muestra la investigación docente realizada por el EICE RIAD, un equipo de innovación docente, de carácter interdisciplinar, cuya finalidad es trabajar competencias transversales entre distintas titulaciones impartidas en la UPV, a partir de un modelo de enseñanza-aprendizaje basado en proyectos.

Se trata por tanto de un ejercicio de caracter generalista pues su interés reside, principalmente, en la estrategia diseñada para enlazar distintas disciplinas, materias y competencias transversales a partir de un proyecto común.

Entre los logros del proyecto está la integración de actividades en las que participan los estudiantes desde las diferentes asignaturas involucradas para la consecución de un objetivo común. La consecución del mismo supone el trabajo cooperativo [García et al. (2001)] entre las áreas de Ingeniería Agronómica y del Medio Natural, Diseño Industrial, Arquitectura, Ingeniería Industrial y Organización de Empresas.

Las asignaturas que confluyen en el proyecto son: Diseño de Bodegas (DdB) de la titulación de Licenciado en Enología que se imparte en la Escuela Técnica Superior de Ingeniería Agronómica del Medio Natural; Diseño básico y creatividad (DBC) de la titulación de Ingeniero Técnico de Diseño Industrial que se imparte en la Escuela Técnica Superior de Ingeniería del Diseño; Fitotecnia General (FG) de la titulación de Ingeniero Agrónomo que se imparte en la Escuela Técnica Superior de Ingeniería Agronómica del Medio Natural; Historia de la Arquitectura III (HQ3) de la titulación de Arquitectura que se imparte en la Escuela Técnica Superior de Arquitectura; Métodos Cuantitativos de ayuda para la toma de decisiones (MCTD) de la Licenciatura de Administración y Dirección de Empresas que se imparte en la Facultad de Administración y Dirección de Empresas; y Planificación Empresarial (RP) del máster Oficial en Dirección y Gestión de proyectos que se imparte en la Escuela Técnica Superior de Ingenieros Industriales.

\section{Objetivos}

El proyecto se ubica en el contexto de Bolonia donde uno de los objetivos estratégicos que persigue la declaración es el incremento del empleo en la Unión Europea (ver http://www.eees.es/pdf/Declaracion_Bolonia.pdf para más información). Por ello, dotar al estudiante de las competencias y habilidades necesarias para integrarse en el mundo laboral constituyen un factor decisivo para la consecución de este objetivo. 
Palomares Figueres, Maite, Ana Portalés Mañanos, Chele Esteve Sendra, Nuria Pascual Seva, MariaVargas Colas, $M^{a}$ Eugenia Babiloni Griñón, Sabina Asensio Ruedas, $M^{a}$ Teresa Sebastiá

Las competencias genéricas y específicas las proporciona cada una de las titulaciones siendo distintas para cada uno de los distintos grados, sin embargo, las competencias transversales pretenden sintetizar el perfil competencial que adquieren todos los alumnos, incluyéndose en el marco de referencia de algunas titulaciones con regulaciones o recomendaciones específicas.

Para la definición de las competencias transversales de la Universitat Politècnica de València se han considerado las normativas y directrices más importantes a nivel nacional e internacional, así como la numerosa literatura científica sobre competencias. El documento de definición de las dimensiones competenciales contempla una relación de 13 conceptos que se despliegan en resultados de aprendizaje para los niveles de grado y máster, siendo las siguientes:

Comprensión e integración, Aplicación y pensamiento práctico, Análisis y resolución de problemas, Innovación, creatividad y emprendimiento, Diseño y proyecto, Trabajo en equipo y liderazgo.Responsabilidad ética, medioambiental y profesional, Comunicación efectiva, Pensamiento crítico, Conocimiento de problemas contemporáneos, Aprendizaje permanente, Planificación y gestión del tiempo, Instrumental específica.

A partir de un proyecto común que englobara distintas disciplinas se trabajaron algunas competencias transversales agrupadas por tareas donde se especificaba que competencias se desarrollaban en cada una de ellas. Se consideró oportuno trabajar con competencias genéricas para aumentar la motivación del alumnado y su implicación con las actividades académicas.

Por otra parte, cabría señalar que el objetivo no fue únicamente implementar tareas sino también evaluar el nivel de consecución de dichas competencias mediante las estrategias que a continuación se detallan.

\section{Desarrollo de la innovación}

Resultó necesario diseñar un proyecto que aglutinara las peculiaridades asociadas a las distintas disciplinas, considerándose óptimo desarrollar el diseño de una bodega, desde un punto de vista global, ya que bajo dicha temática además de reunir las diversas disciplinas, el caso de estudio podía convertirse en un proyecto real.

Definido el objetivo se implementaron sucesivos proyectos de innovación y mejora educativa (PIME) como estrategia docente.

También hay que recalcar que este tipo de docencia requería un equipo de investigación docente interdisciplinar cuyos componentes desarrollaran su actividad en diferentes titulaciones. Cabe destacar que algunos de los profesores que conformaban el equipo tenían 
contacto directo con las necesidades reales de los proyectos pues compatibilizaban la docencia con su actividad profesional. Por otra parte, todos los profesores miembros del proyecto habian participado en el Programa de Formación Inicial Pedagógica para el Profesorado Universitario (FIPPU). La formación adquirida a través de este programa, las experiencias docentes compartidas, junto con la problemática identificada impulsaron la innovación docente propuesta.

1.1 PIME 2010-2011. “desarrollo de una metodología de enseñanza-aprendizaje orientada hacia la adquisición de competencias mediante el desarrollo de proyectos multidisciplinares”

El primer proyecto se encaminó a sentar las bases metodológicas de esta nueva forma de desarrollo de proyectos multidisciplinares, y aplicarlas para detectar la capacidad de integrar de manera adecuada a equipos de trabajo de alumnos de diferentes áreas. Para lograr dicho objetivo se diseñó un espacio de conocimiento integrado, a través del planteamiento de diferentes actividades y metodologías en asignaturas de distintas titulaciones (ingenierías y arquitectura). Esta era una manera de poner en práctica metodologías de Enseñanza-Aprendizaje en el marco de un proyecto común.

Las tareas y metodologías docentes se detallan a continuación y se organizan por asignaturas:

Tarea 0. Las profesoras coordinadoras del proyecto crearon un blog para poner en común los resultados de las diferentes Tareas. Dicho trabajo se realizó a través de la herramienta disponible en web de la Universidad Politécnica de Valencia (www.poliblog.upv.es). Dicho blog se utilizó como repositorio de los trabajos de los alumnos participantes en el proyecto y tanto las profesoras como los alumnos introdujeron comentarios sobre las distintas experiencias realizadas.

Tareas 1 y 2. Diseño de Bodegas (DdB): mediante trabajos en grupo y aprendizaje basado en proyectos se abordaron los aspectos relativos al emplazamiento de la bodega, la selección de variedades de uva, la ingeniería del proceso de elaboración, el cálculo de instalaciones y el diseño en planta.

Tarea 3. Diseño básico y creatividad (DBC): a través del aprendizaje basado en proyectos se trabajó en el diseño de la botella y de la etiqueta para los vinos que se elaborados según resultados de la Tareas 1 y 2 .

Tarea 4. Planificación Empresarial (RP): mediante un trabajo individual tutorizado el 
Palomares Figueres, Maite, Ana Portalés Mañanos, Chele Esteve Sendra, Nuria Pascual Seva, MariaVargas Colas, $M^{a}$ Eugenia Babiloni Griñón, Sabina Asensio Ruedas, $M^{a}$ Teresa Sebastiá

alumno seleccionó el software necesario para la gestión integral de las bodegas proyectadas en la Tareas 1 y 2.

Tarea 6. Historia de la Arquitectura III (HQ3): los alumnos, en grupos de trabajo, analizaron los proyectos de bodegas realizados en España por arquitectos de prestigio nacional e internacional incluyendo los parámetros de diseño obtenidos en la Tarea 2.

Tarea 7. Fitotecnia General (FG): mediante la técnica del puzle de Aronsen, se calcularon las necesidades hídricas y nutritivas de viñas, en función de su emplazamiento y las variedades cultivadas (escogidas por en la Tarea 1 ).

Tarea 8. Métodos cuantitativos en la ayuda a la toma de decisiones (MCTD): se utilizó como metodología docente el Estudio de Casos resolviendo casos prácticos relacionados con las decisiones estratégicas tomar en relación con las Tareas 2 y 7: momento óptimo de vendimia, volumen destinado a granel, volumen a embotellar.

1.2 PIME 2011-2012 “desarrollo y evaluación de competencias transversales mediante metodologías de enseñanza-aprendizaje interdisciplinares”

Para el segundo PIME, se diseñaron tareas que a diferencia del año anterior aglutinaban a distintas asignaturas. En cada tarea se señalaban las competencias transversales a trabajar así como las profesoras responsables del seguimiento y el periodo de impartición. Se elaboró una tabla en la que se sincronizaban las distintas tareas $\mathrm{Y}$ se elaboraron cuestionarios para cada asignatura que permitían valorar en una escala de 1 a 4 el grado de consecución de cada competencia.

Tarea 1. Selección de un sistema integrado de gestión

Competencias genéricas: Trabajo en equipo; capacidad de análisis y de síntesis; comunicación escrita; capacidad de gestión de la información; toma de decisiones; uso de las TICs; pensamiento crítico; pensamiento práctico

Responsables: Maria Vargas (DDB) - Sabina Asensio (ERP)

Calendario: Cuatrimestre A

Los alumnos de la asignatura Planificación Empresarial (ERP), organizados en grupos, recabaron la información necesaria para llevar a cabo la selección del sistema integrado de gestión más adecuado a la bodega que habian diseñando los alumnos deDiseño de Bodegas (DDB). Para ello, los alumnos de ERP elaboraron un cuestionario para recopilar

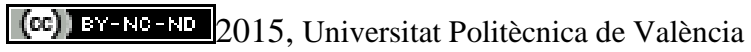

Congreso In-Red (2015) 
información para la selección adecuada del sistema de gestión. Dichos formularios fueron publicados en el blog RIAD Bodegas y fueron cumplimentados por los alumnos de DDB, organizados también en grupos. Los alumnos de ERP elaboraron un informe en el que estudiaron al menos cuatro sistemas integrados de gestión del mercado justificando su adaptación y adecuación a la bodega diseñada por los alumnos de DDB. Finalmente, seleccionaron la mejor solución. El informe de selección fue publicado en el blog RIAD_bodegas y revisado por los alumnos de DDB. Se evaluó, mediante rúbricas, la calidad de los cuestionarios elaborados, así como las respuestas proporcionadas por los alumnos. También se evaluó la utilidad final para la selección del sistema de gestión de la información requerida y obtenida.

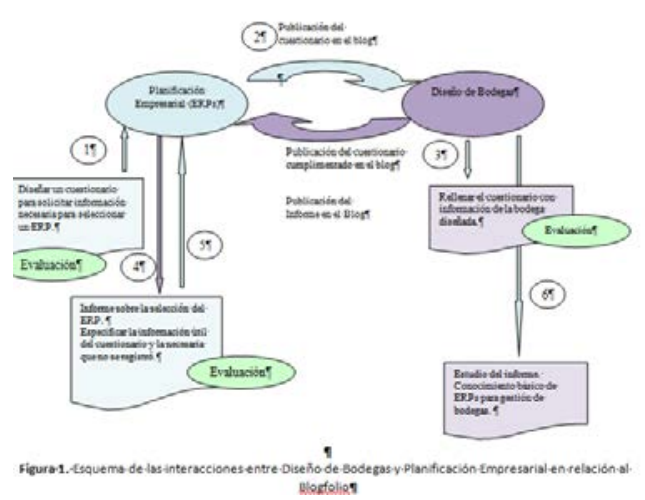

Tarea 2. Diseño agronómico del riego de viñas

Competencias genéricas: Trabajo en equipo, comunicación oral, pensamiento crítico

Responsables: Maria Vargas (DDB) - Núria Pascual (FG)

Calendario: Cuatrimestre A

Los alumnos de la asignatura Fitotecnia General (FG) realizaron un trabajo sobre el diseño agronómico del riego del viñedo. Para ello obtuvieron los datos necesarios para el diseño (localización de la viña, variedad, edad de la plantación, etc.), a través de cuestionarios publicados en el blog RIAD_bodegas y que fueron contestados por los alumnos de la asignatura de DDB. Con la información recabada los alumnos de FG realizaron una búsqueda bibliográfica de los datos necesarios para el diseño agronómico (datos climáticos, coeficientes de cultivo, criterios de riego, etc.). Se evaluó la calidad de las preguntas planteadas en el cuestionario así como el número y fiabilidad de las fuentes contrastadas Finalmente, los alumnos de FG elaboraron un informe sobre el diseño agronómico del sistema de riego y que se publicó en el blog para que todos los alumnos participantes en la innovación pudiesen consultarlo y comentarlo. 
Palomares Figueres, Maite, Ana Portalés Mañanos, Chele Esteve Sendra, Nuria Pascual Seva, MariaVargas Colas, $M^{a}$ Eugenia Babiloni Griñón, Sabina Asensio Ruedas, $M^{a}$ Teresa Sebastiá

Tarea 3. Estrategias de promoción del vino español en el mercado chino.

Competencias genéricas: Aprendizaje autónomo; comunicación oral y escrita en Inglés; toma de decisiones; capacidad de gestión de la información; capacidad de planificación y organización; comprensión de otras culturas y costumbres; resolución de problemas; trabajo en equipo; creatividad; uso de las TICs.

Responsable: Chele Esteve Sendra

Se diseñó una actividad de trabajo en equipo donde los alumnos de la asignatura New Promotional Platforms analizaron y crearon una estrategia de promoción de un vino valenciano en el mercado chino mediante nuevas plataformas. Para cumplir con su objetivo, los alumnos estudiaron modelos de lanzamiento de bebidas alcohólicas en China y analizaron la demanda. Para facilitar el desarrollo de la actividad en cuanto al análisis del mercado, se contó con la colaboración de la Oficina del ICEX en Shanghái y con el apoyo y experiencia de una bodega valenciana. Se facilitó a los alumnos métodos de previsión de demanda, que fueron necesarios para seleccionar la estrategia de promoción es más adecuada así como realizar previsiones sobre las ventas futuras. En relación a esta tarea se evaluó el proyecto de lanzamiento en sus diferentes fases y el resultado final fue presentado en el aula y publicado en el blog RIAD-Bodegas.

Tarea 4. Criterios para el estudio del funcionamiento de bodegas

Competencias genéricas: Capacidad de análisis, capacidad de gestión de la información, trabajo en equipo; pensamiento crítico.

Asignaturas: Urbanística 1 (URB 1) e Historia de la Arquitectura 3 (HQ3)

Responsables: Ana Portalés Mañanós y Maite Palomares Figueres.

Los alumnos de DDB, en equipos de trabajo, diseñaron una bodega estableciéndose y cuantificándose los parámetros necesarios y apropiados para llevar a cabo dicha actividad y redactaron un pre-diseño/anteproyecto que fue facilitado a los alumnos de Historia de la Arquitectura III (HQ3). Los alumnos de HQ3 contrastaron los documentos facilitados por los alumnos de DDB con los resultados obtenidos en su análisis de bodegas emblemáticas con el objetivo de elaborar una guía para el desarrollo de futuras experiencias similares. Algunos de los trabajos han sido publicados en el blog RIAD_Bodegas.

1.3 PIME 2012-2013. "El blog como portafolio virtual de un proyecto interdisciplinar"

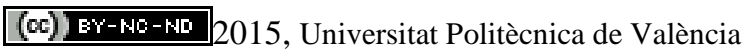

Congreso In-Red (2015) 
Tras la experiencia adquirida durante la realización de los proyectos de los años anteriores, se planteó potenciar y mejorar el uso del Blog como herramienta vertebradora del proyecto común de diseño de una Bodega. Si bien dicha herramienta ya había sido empleada en los proyectos de innovación desarrollados hasta el momento, se consideró necesario un estudio más profundo de aspectos tales como: estructura; mecanismos de edición y mantenimiento del blog por parte de los alumnos, profesores; así como establecer mecanismos que permitieran una evaluación exhaustiva del trabajo realizado por los alumnos en relación con el contenido del blog. Además se pretendía introducir un nuevo apartado para potenciar la relación alumno-empresa. Este nuevo apartado empresarial del blog se diseñó con un doble carácter: docente, al tratarse de una herramienta para la enseñanza-aprendizaje, y también cabría considerar su carácter profesional, reflejando una intención de acercamiento al mundo laboral. La sección del blog destinada a las “relaciones con la empresa” se centró en la profesionalización del alumnado y presentaba dos partes claramente diferenciadas y destinadas cada una de ellas al alumno o a la empresa. En su diseño, esta sección contaba con todos los elementos de caracterización de un blog profesional (botones sociales, llamadas a la acción, enlaces a webs) buscando una correspondencia con la realidad y la máxima participación de las empresas participantes. De este modo el espacio virtual diseñado reflejaba del mundo laboral incrementando las posibles sinergias existentes.

A las tareas desarrolladas los años anteriores se añadieron dos nuevas y además se completaron las que ya habían sido puestas en marcha, incidiendo en la reunión de varias asignaturas.

Tarea 5. Gestión de Recursos Humanos (RRHH)

Competencias genéricas: Trabajo en equipo, pensamiento crítico, gestión de la información, comunicación oral y escrita, aplicación de conocimientos teóricos a la práctica, planificación eficiente del trabajo, capacidad de síntesis.

Titulación: Grado en Gestión y Administración Pública

Escuela/Facultad: Facultad de Administración y Dirección de Empresas

Responsable: Eugenia Babiloni

La tarea consistió en la realización el análisis y descripción de un puesto de trabajo. El análisis y descripción de un puesto de trabajo es un proceso sistemático de estudio que permite concretar y reflejar documentalmente la naturaleza, contenido y alcance de cada posición funcional dentro de una organización. Este análisis consta fundamentalmente de dos partes: (1) Análisis del puesto a partir de una recogida sistemática de la información relevante; y (2) Descripción del puesto en un documento apropiado. 
Palomares Figueres, Maite, Ana Portalés Mañanos, Chele Esteve Sendra, Nuria Pascual Seva, MariaVargas Colas, $M^{a}$ Eugenia Babiloni Griñón, Sabina Asensio Ruedas, $M^{a}$ Teresa Sebastiá

Es importante incluir los niveles de actitud, conocimiento, habilidades y experiencia necesarios para poder desempeñar el puesto, la responsabilidad que se le va a exigir al ocupante del mismo, el entorno físico donde se desarrollará, etc.

La tarea se realizó en grupo y los puestos de trabajo analizados fueron el de enólogo de una bodega. Para ello, a los alumnos se les facilitó una descripción básica de los puestos a través del blog.

INPUTS del BLOG: Descripción básica de las funciones realizadas por un enólogo en una bodega. Esta descripción estaba disponible en el blog y fue facilitada por María Vargas (DDB) y Nuria Pascual (FG). Además el alumno utilizó el blog como herramienta para recabar información, ponerse en contacto con otros alumnos involucrados en el proyecto, profesores, e incluso profesionales del sector.

OUTPUTS al BLOG: Descripción y análisis del puesto de enólogo realizado por los alumnos en el tiempo y forma establecidos. Presentación del documento normalizado con la tarea de recogida de información resultante del proyecto así como la evaluación de la tarea dentro del mismo (Tarea 1).
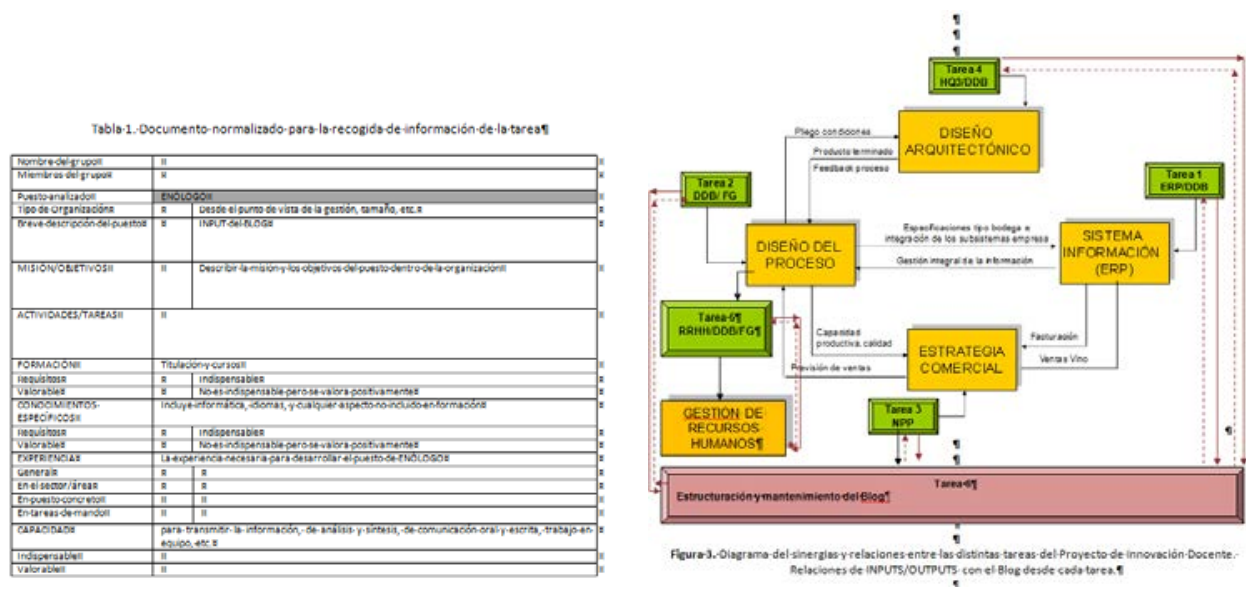

Tarea 6. Estructuración mantenimiento del Blog

Competencias: trabajo en equipo, pensamiento crítico, uso de las TICs

Responsables: Todas las profesoras participantes en el Proyecto de Innovación

Calendario: Cuatrimestre A y B.

Partiendo del blog se diferenciaban dos partes: la destinada a presentar las aportaciones del alumnado y la reservada a responder a las demandas empresariales del sector del vino. Los alumnos presentaron, además de los inputs solicitados en las Tareas descritas para cada

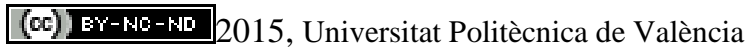

Congreso In-Red (2015) 
asignatura, una selección de propuestas innovadoras que relacionaban las asignaturas que cursadas con el sector bodeguero. Posteriormente los profesores realizaron una selección de dichas propuestas que fueron colgadas en el apartado dedicado a las empresas.

En el apartado del blog destinado a empresas se introdujo una relación de las más significativas vinculadas al vino, seleccionando e informando sobre sus aportaciones más recientes.

Considerando que el éxito del blog reside en fomentar de las interacciones que propicia la propia herramienta del blog, se requería una necesaria puesta al día y mantenimiento de los contenidos y sugerencias. Para conseguirlo fue decisivo que en la evaluación de la actividad repercutiera, ampliamente, el estado en que se encuentre la actualización de la información contenida en este apartado.

\section{Resultados}

Podemos valorar los resultados obtenidos agrupados en tres categorías, a partir de las evaluaciones:

\section{Evaluación de competencias transversales:}

En cada una de las asignaturas implicadas, una parte de la evaluación se centró en el nivel de consecución de las competencias transversales planteadas a través de las tareas previstas en el proyecto. Se elaboraron cuestionarios por asignaturas que valoraban en una escala de 1 a 4 el grado de consecución de cada competencia. Un ejemplo de cuestionario para evaluar la competencia Comunicación Escrita, competencia transversal común a todas las tareas, sería el siguiente (Tabla 2).

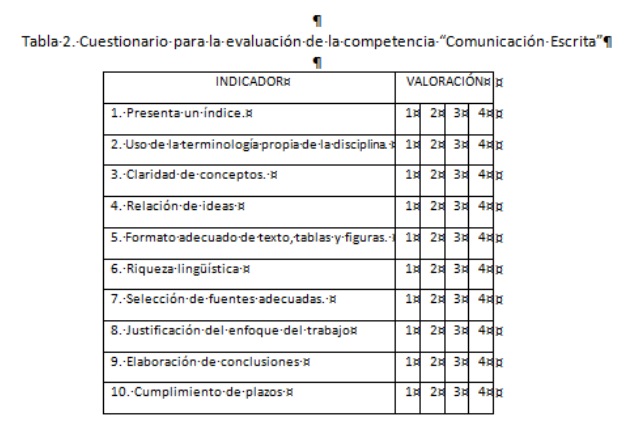

También se analizó el rendimiento de los alumnos en cada una de las asignaturas implicadas en el proyecto en comparación con el histórico de cursos académicos en los que 
Palomares Figueres, Maite, Ana Portalés Mañanos, Chele Esteve Sendra, Nuria Pascual Seva, MariaVargas Colas, $M^{a}$ Eugenia Babiloni Griñón, Sabina Asensio Ruedas, $M^{a}$ Teresa Sebastiá

no se evaluaban explícitamente las competencias transversales. Así, se observa en las fichas elaboradas por los alumnos de HQ3

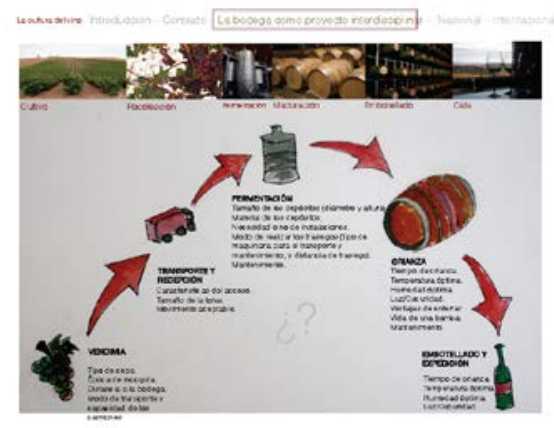

\section{Evaluación del uso del blogfolio en todas las asignaturas:}

En relación al empleo del Blog como portafolio del proyecto, en cada una de las asignaturas involucradas, los alumnos (individualmente o en equipos de un máximo de 3 personas) prepararon al menos una contribución semanal al blog (con posts o comentarios) en relación a cada una de las tareas involucradas.

Cada uno de los profesores facilitó a los alumnos de su asignatura una plantilla-guía, con la estructura y los aspectos a considerar.
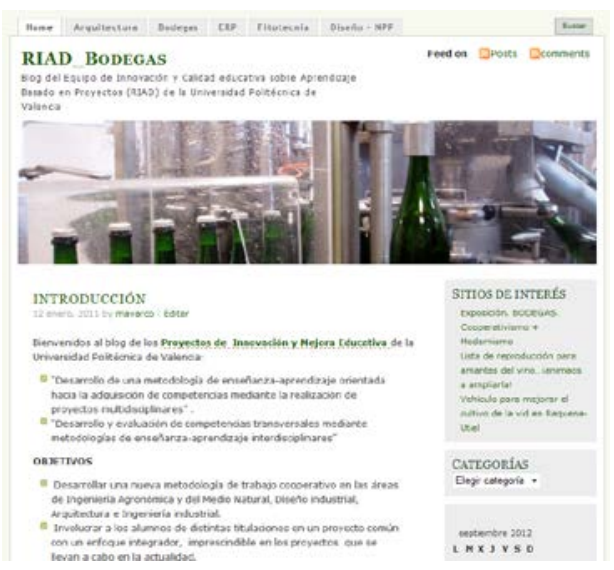

\section{Evaluación de la innovación:}

\section{(cc) EY-Nc-ND 2015, Universitat Politècnica de València}


RIAD. La experiencia de los PIME's aplicada a un aprendizaje basado en proyectos

En las asignaturas participantes en el Proyecto, los alumnos respondieron una encuesta de opinión (disponible en PoliformaT), donde indicaban su grado de desacuerdo o de acuerdo en relación a las siguientes afirmaciones en una escala de 0 a 7 :

He escrito al menos un post en el blog cada semana; Los contenidos de mis posts han sido copiados directamente de la fuentes consultadas (directamente un "copy-paste”); Los comentarios generados por los posts me parecen adecuados; El diseño del blog me ha facilitado la participación en el mismo ; El contenido del blog me ha facilitado la comprensión de las tareas asignadas; La información que he aportado en mis comentarios y posts procede directamente de la web ; La información que he aportado en mis comentarios y posts procede de la bibliografía recomendada en la asignatura.; Los posts y comentarios me han aportado información que desconocía; Seguiré el blog en el futuro; Colaboraré en el blog en el futuro

Además, se cuantificó el número de posts en el blog y de comentarios semanales en relación a cada una de las secciones en las que se articula el blog, teniendo en cuenta si procedían de un alumno, de un profesor, o de alguien externo a la Universidad.

\section{Conclusiones}

La innovación realizada ha sido muy enriquecedora tanto para los alumnos como para las profesoras involucradas. La experiencia es trasladable a otros contextos (titulaciones y asignaturas de la UPV), teniendo siempre presente que requiere de una gran voluntad por parte de los docentes y alumnos implicados, un gran nivel de compromiso e implicación y que es necesario, como primer paso, el diseño de un proyecto atractivo y capaz de involucrar, tanto por su actualidad temática como por su viabilidad, a distintas titulaciones y materias.

El blog se ha mostrado como una herramienta eficaz para la interrelación y comunicación entre los participantes en el PIME así como para la difusión de la innovación. No obstante, se han detectado aspectos a mejorar en relación al blog. Así por ejemplo, sería conveniente conseguir una estructura más cohesionada e uniforme con el fin de facilitar la recogida de información y el análisis de los resultados. Por otra parte, para que sea un verdadero elemento vertebrador, el blog necesita de una mayor participación de los alumnos. Para conseguir esta participación, se debe valorar (y evaluar) su participación, tanto en cantidad como en calidad de las entradas realizadas en el blog. Todas estas propuestas se materializaron en un nuevo PIME donde se pretendía mantener el esquema de sinergias entre asignaturas ya establecido y consolidado y convertir el blog en un "blogfolio" para conseguir un espacio en el que se recogieran evidencias sobre la adquisición de competencias transversales de los alumnos en relación a cada una de las tareas. Dicho 
Palomares Figueres, Maite, Ana Portalés Mañanos, Chele Esteve Sendra, Nuria Pascual Seva, MariaVargas Colas, $M^{a}$ Eugenia Babiloni Griñón, Sabina Asensio Ruedas, $M^{a}$ Teresa Sebastiá

PIME fue denegado, sin embargo, creemos e la oportunidad de dicha propuesta y a continuación mostramos algunas de las imágenes publicadas en el blog que plasman los resultados obtenidos y la vinculación entre las distintas tareas, en el ejemplo siguiente Tarea 2 y 4.
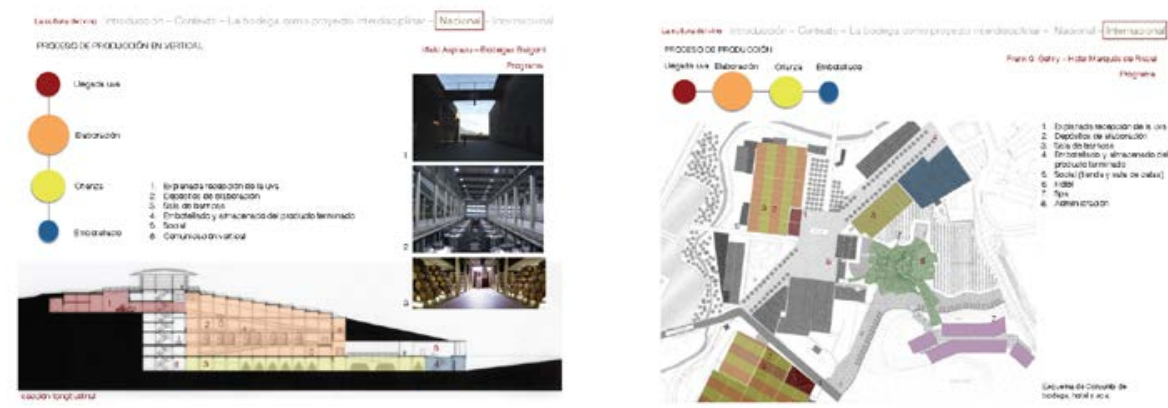

\section{Referencias}

DINOV, I.D. (2008). Integrated, multidisciplinary and technology-enhanced science education: the next frontier. MERLOT Journal of online learning and teaching, 4 (1): 8493.

GARCÍA, R., TRAVER, J. A., CANDELA, I. (2001). Aprendizaje cooperativo. Fundamentos, características y técnicas. Madrid: CCS.

TRILLO M.A., ESPEJO R., GÁMEZ C. M. (2009). A multidisciplinary experience in the European context. Technology, education and development (pp. 217-228). Croatia.

TEKISNARLAN, E. ( 2008). Blogs: a qualitative investigation into an instructor and undergraduate students' experiences. Australasian Journal of educational technology, 24 (4): 402-412.

YÁNIZ C., VILLARDÓN L. (2006). Planificar desde competencias para promover el aprendizaje. Cuadernos Monográficos del ICE, núm. 12. Bilbao: Universidad de Deusto. 